STRUCTURAL SCIENCE CRYSTAL ENGINEERING MATERIALS

ISSN 2052-5206

Received 31 March 2021

Accepted 9 August 2021

Edited by J. Lipkowski, Polish Academy of Sciences, Poland

Keywords: magnetic structure; antiferromagnetism; neutron diffraction; magnetometric measurements; symmetry analysis.

Supporting information: this article has supporting information at journals.iucr.org/b

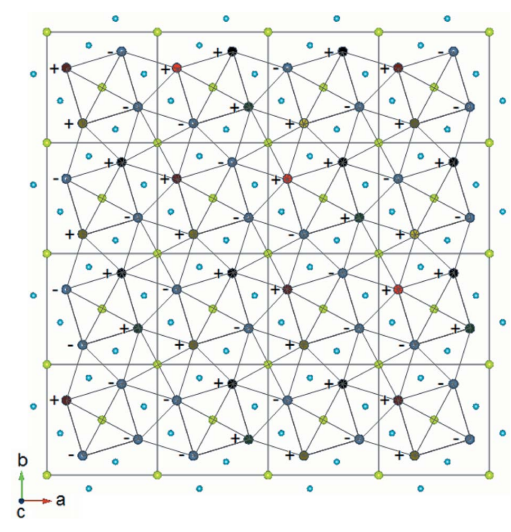

OPEN ○ ACCESS

\section{Crystal and magnetic structures of $R_{2} \mathrm{Ni}_{1.78} \mathrm{In}$ compounds $(R=\mathrm{Tb}, \mathrm{Ho}$, Er and $\mathrm{Tm})$}

\author{
Stanisław Baran, ${ }^{\text {a* }}$ Aleksandra Deptuch, ${ }^{\mathrm{b}}$ Andreas Hoser, ${ }^{\mathrm{c}}$ Bogusław Penc, ${ }^{\mathrm{a}}$ \\ Yuriy Tyvanchuk ${ }^{\mathrm{d}}$ and Andrzej Szytuła ${ }^{\mathrm{a}}$
}

\begin{abstract}
${ }^{a}$ M. Smoluchowski Institute of Physics, Jagiellonian University, Prof. Stanisława Łojasiewicza 11, PL-30-348 Kraków, Poland, ' Institute of Nuclear Physics, Polish Academy of Sciences, Radzikowskiego 152, PL-31-342 Kraków, Poland,

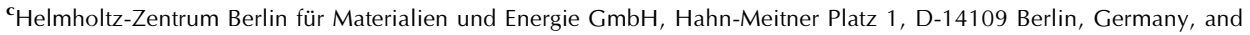
dAnalytical Chemistry Department, Ivan Franko National University of Lviv, Kyryla i Mefodiya 6, 79005 Lviv, Ukraine. *Correspondence e-mail: stanislaw.baran@uj.edu.pl
\end{abstract}

The crystal and magnetic structures in $R_{2} \mathrm{Ni}_{1.78} \mathrm{In}(R=\mathrm{Ho}, \mathrm{Er}$ and $\mathrm{Tm})$ have been studied by neutron diffraction. The compounds crystallize in a tetragonal crystal structure of the $\mathrm{Mo}_{2} \mathrm{FeB}_{2}$ type (space group $\mathrm{P} 4 / \mathrm{mbm}$ ). At low temperatures, the magnetic moments, localized solely on the rare earth atoms, form antiferromagnetic structures described by the propagation vector $\mathbf{k}=\left[k_{x}\right.$, $\left.k_{x}, \frac{1}{2}\right]$, with $k_{x}$ equal to $\frac{1}{4}$ for $R=\mathrm{Er}$ and Tm or 0.3074 (4) for $R=$ Ho. The magnetic moments are parallel to the $c$ axis for $R=$ Ho or lie within the (001) plane for $R=\mathrm{Er}$ and Tm. The obtained magnetic structures are discussed on the basis of symmetry analysis. The rare earth magnetic moments, determined from neutron diffraction data collected at $1.6 \mathrm{~K}$, are $6.5(1) \mu_{\mathrm{B}}(\mathrm{Er})$ and 6.09 (4) $\mu_{\mathrm{B}}$ $(\mathrm{Tm})$, while in the incommensurate modulated magnetic structure in $\mathrm{Ho}_{2} \mathrm{Ni}_{1.78} \mathrm{In}$ the amplitude of modulation of the Ho magnetic moment is $7.93(8) \mu_{\mathrm{B}}$. All these values are smaller than those expected for the respective free $R^{3+}$ ions. A symmetry analysis of the magnetic structure in $\mathrm{Tb}_{2} \mathrm{Ni}_{1.78} \mathrm{In}$ is also included, as such information is missing from the original paper [Szytuła, Baran, Hoser, Kalychak, Penc \& Tyvanchuk (2013). Acta Phys. Pol. A, 124, 994-997]. In addition, the results of magnetometric measurements are reported for $\mathrm{Tm}_{2} \mathrm{Ni}_{1.78} \mathrm{In}$. The compound shows antiferromagnetic ordering below the Néel temperature of $4.5 \mathrm{~K}$. Its magnetic properties are found to originate from magnetic moments localized solely on the thulium atoms (the nickel atoms remain non-magnetic in $\left.\mathrm{Tm}_{2} \mathrm{Ni}_{1.78} \mathrm{In}\right)$. The reduction of rare earth magnetic moments in the ordered state in $R_{2} \mathrm{Ni}_{1.78} \mathrm{In}(R=\mathrm{Tb}, \mathrm{Ho}, \mathrm{Er}$ and $\mathrm{Tm})$ and the change in direction of the moments indicate the influence of the crystalline electric field $(\mathrm{CEF})$ on the stability of the magnetic order in the investigated compounds.

\section{Introduction}

Intermetallic compounds of the $R-\mathrm{Ni}-\mathrm{In}$ systems are the subject of intensive studies with respect to their crystal structures, chemical bonds and magnetic properties (Kalychak et al., 2004). Within these systems, the compounds with a stoichiometry close to 2:2:1 have been observed to crystallize in two different crystal structures:

(i) A tetragonal one of the $\mathrm{Mo}_{2} \mathrm{FeB}_{2}$ type (space group $P 4 / \mathrm{mbm})$ found for both the $R_{2} \mathrm{Ni}_{2} \mathrm{In}(R=\mathrm{La}-\mathrm{Nd})$ stoichiometric composition and the $R_{2} \mathrm{Ni}_{2-x}$ In $(x=0.22, R=\mathrm{Y}, \mathrm{Sm}$, Gd-Tm, Lu) nonstoichiometric composition (Kalychak et al., 1990).

(ii) An orthorhombic one of the $\mathrm{Mn}_{2} \mathrm{AlB}_{2}$ type (space group Cmmm) found exclusively for the $R_{2} \mathrm{Ni}_{2} \mathrm{In}(R=\mathrm{Y}, \mathrm{Sm}$, 
Gd-Tm, Lu) stoichiometric composition (Kalychak et al., 1990; Zaremba et al., 1988).

The $R_{2} \mathrm{Ni}_{2-x}$ In $(R=\mathrm{Gd}-\mathrm{Er})$ nonstoichiometric compounds order antiferromagnetically below the Néel temperatures $\left(T_{\mathrm{N}}\right)$ of $26.4 \mathrm{~K}(\mathrm{Gd}), 20.2 \mathrm{~K}(\mathrm{~Tb}), 8.3 \mathrm{~K}(\mathrm{Dy}), 7.1 \mathrm{~K}(\mathrm{Ho})$ and $6.3 \mathrm{~K}$ (Er) (Tyvanchuk et al., 2012).

Studies of the physical properties of $R_{2} \mathrm{Ni}_{2}$ In reveal that $\mathrm{Ce}_{2} \mathrm{Ni}_{2} \mathrm{In}$ is a non-magnetic intermediate-valence system (Hauser et al., 1997; Kaczorowski et al., 1996), while $\mathrm{Nd}_{2} \mathrm{Ni}_{2} \mathrm{In}$ orders antiferromagnetically below $8 \mathrm{~K}$ (Maskova et al., 2014). Magnetic and thermodynamic studies of $R_{2} \mathrm{Ni}_{2} \mathrm{In}(R=\mathrm{Gd}-$ $\mathrm{Tm})$ report an antiferromagnetic order with Néel temperatures between $5 \mathrm{~K}(\mathrm{Tm})$ and $40 \mathrm{~K}$ (Tb) (Szytuła et al., 2015).

Neutron diffraction data confirm the antiferromagnetic ground state in selected members of both stoichiometric and nonstoichiometric families of compounds. The antiferromagnetic structure corresponds to the following propagation vectors: $\mathbf{k}=\left[\frac{1}{4}, \frac{1}{4}, \frac{1}{2}\right]$ in $\mathrm{Tb}_{2} \mathrm{Ni}_{1.78} \mathrm{In},\left[\frac{1}{2}, \frac{1}{2}, \frac{1}{2}\right]$ in $\mathrm{Tb}_{2} \mathrm{Ni}_{2} \mathrm{In}$ (Szytuła et al., 2013) and $\left[\frac{1}{2}, 0, \frac{1}{2}\right]$ in $R_{2} \mathrm{Ni}_{2} \mathrm{In}(R=\mathrm{Er}, \mathrm{Tm})$ (Baran et al., 2017).

A recent paper on magnetism in the 2:2:1 compounds reports a ferromagnetic order for $R=\operatorname{Pr}$ and $\mathrm{Nd}$, and an antiferromagnetic order for $R=$ Dy and Ho. A considerable magnetocaloric effect is detected in the vicinity of the magnetic transition in all four compounds (Zhang et al., 2019).

This work is a continuation of our investigation of the magnetic properties of the compounds belonging to the $R-\mathrm{Ni}-$ In systems. We report here for the first time the lowtemperature magnetic structures of $R_{2} \mathrm{Ni}_{1.78} \mathrm{In}(R=\mathrm{Ho}, \mathrm{Er}$, $\mathrm{Tm})$ as determined from powder neutron diffraction. The validity of the structures is verified by symmetry analysis. In addition, we present a symmetry analysis of the $\mathrm{Tb}_{2} \mathrm{Ni}_{1.78} \mathrm{In}$ magnetic structure which was missing from the original paper (Szytuła et al., 2013). The magnetic properties of $\mathrm{Tm}_{2} \mathrm{Ni}_{1.78} \mathrm{In}$, as derived from DC magnetic measurements, are also reported.

\section{Experimental details}

Polycrystalline samples of $R_{2} \mathrm{Ni}_{1.78} \mathrm{In}(R=\mathrm{Ho}$, Er and Tm $)$ of a total weight of $5 \mathrm{~g}$ each were obtained by arc melting of the constituent elements (purity $99.9 \mathrm{wt} \%$ or better) under an argon atmosphere. The obtained ingots were encapsulated in evacuated silica tubes and annealed at $870 \mathrm{~K}$ for one month, then quenched in cold water. Finally, the samples' quality was checked by X-ray powder diffraction performed at room temperature using a PANalytical X'Pert PRO diffractometer ( $\mathrm{Cu} K_{\alpha}$ radiation).

DC magnetic measurements were carried out using a commercial vibrating sample magnetometer (VSM) option installed on the PPMS platform by Quantum Design. The data were collected in the temperature range $2-300 \mathrm{~K}$ in magnetic fields up to $90 \mathrm{kOe}$.

Powder neutron diffraction patterns were taken at low temperature $(1.6 \mathrm{~K}$ ) and in the paramagnetic state (above the respective Néel temperature) on the E6 diffractometer at
Table 1

Phase compositions (wt \%) of the investigated $R_{2} \mathrm{Ni}_{1.78} \mathrm{In}$ samples.

\begin{tabular}{llllc}
\hline & \multicolumn{1}{l}{$R$} & & \\
\cline { 2 - 5 } Phase & $\mathrm{Tb}$ & Ho & $\mathrm{Er}$ & $\mathrm{Tm}$ \\
\hline $2: 1.78: 1$ & $84.8(9)$ & $88.0(1.4)$ & $76.9(1.0)$ & $80.3(1.8)$ \\
$2: 2: 1$ & $15.2(9)$ & & & $6.6(8)$ \\
$5: 2: 4$ & & $12.0(1.4)$ & $16.8(2.3)$ & $10.8(1.4)$ \\
$R_{2} \mathrm{O}_{3}$ & & & $6.3(5)$ & $2.4(5)$ \\
\hline
\end{tabular}

Helmholtz-Zentrum Berlin für Materialien und Energie $\mathrm{GmbH}$. The incident neutron wavelength was $2.432 \AA$.

For Rietveld analysis of X-ray and neutron diffractograms the computer program FULLPROF was utilized (RodríguezCarvajal, 1993, 2001), while for symmetry analysis the computer program BASIREPS, which is distributed together with FULLPROF, was used.

No new data were collected for the $\mathrm{Tb}_{2} \mathrm{Ni}_{1.78} \mathrm{In}$ sample. The previously reported neutron diffraction patterns (Szytuła et al., 2013) were re-analyzed on the basis of symmetry analysis.

\section{Results}

\subsection{Crystal structure}

The X-ray diffraction data collected at room temperature confirm the previously reported tetragonal crystal structure of the $\mathrm{Mo}_{2} \mathrm{FeB}_{2}$ type. Detailed analysis of the powder neutron diffraction patterns taken in the paramagnetic state provided information on both the samples' phase composition and their crystal structure parameters. The investigated samples contain about $80 \%$ of the $R_{2} \mathrm{Ni}_{1.78}$ In phase with a small content of the 2:2:1 and 5:2:4 phases. In addition, in the Er- and Tm-based samples small amounts of the $R_{2} \mathrm{O}_{3}$ oxides were detected (see Table 1).

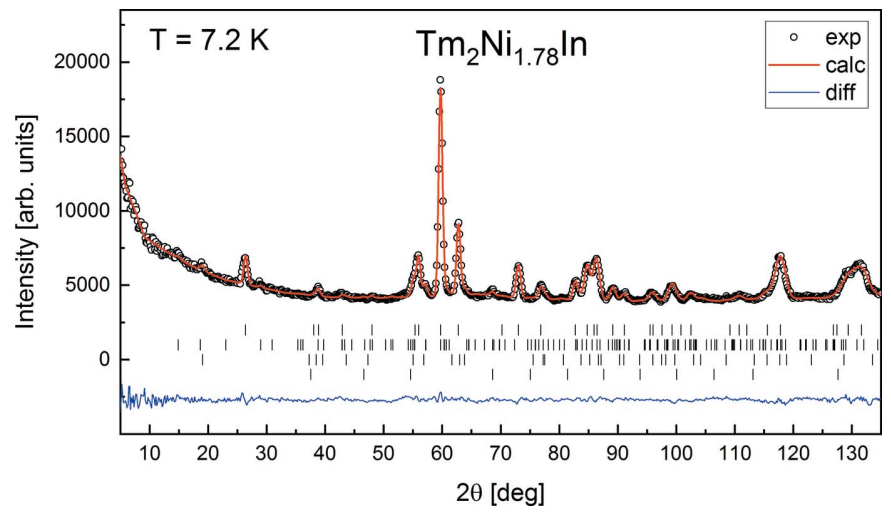

Figure 1

The neutron diffraction pattern of $\mathrm{Tm}_{2} \mathrm{Ni}_{1.78} \mathrm{In}$ collected at $T=7.2 \mathrm{~K}$ in the paramagnetic state (open circles), together with the results of the Rietveld refinement (red line) and the difference curve (bottom line). The first row of vertical bars refers to the peak positions originating from the $\mathrm{Tm}_{2} \mathrm{Ni}_{1.78}$ In main phase [80.3 (1.8) wt\%], while the second, third and fourth rows correspond to reflections from the $\mathrm{Tm}_{5} \mathrm{Ni}_{2} \mathrm{In}_{4}$ (Szytuła et al., 2014) [10.8 (1.4) wt\%], $\mathrm{Tm}_{2} \mathrm{Ni}_{2} \mathrm{In}$ (Baran et al., 2017) [6.6 (8) wt\%] and $\mathrm{Tm}_{2} \mathrm{O}_{3}$ (Maslen et al., 1996) [2.4 (5) wt\%] impurity phases, respectively. 
Table 2

Crystal structure parameters for $R_{2} \mathrm{Ni}_{1.78} \mathrm{In}(R=\mathrm{Tb}, \mathrm{Ho}$, Er, Tm; space group $P 4 / \mathrm{mbm})$ determined by Rietveld refinement from neutron diffraction patterns collected in the paramagnetic state.

For $R=\mathrm{Tb}$, Er the nearest-neighbour distances $(\mathrm{NN})$ are between atoms $R 1$ and $R 4$ and between atoms $R 2$ and $R 3$, and the next-nearest neighbour distance $(\mathrm{NNN})$ is equal to the $c$ lattice parameter. For $R=\mathrm{Ho}, \mathrm{Tm}$ there is a reverse relationship: $\mathrm{NN}=c$ and NNN $=R 1-R 4$ and $R 2-R 3$ distances.

\begin{tabular}{|c|c|c|c|c|c|c|c|c|}
\hline \multirow[b]{2}{*}{ Parameter } & \multicolumn{8}{|l|}{$R / T(\mathrm{~K})$} \\
\hline & $\mathrm{Tb} / 29.9$ & $\mathrm{~Tb} / 1.6$ & Ho/12.2 & Ho/1.6 & $\mathrm{Er} / 12.0$ & $\mathrm{Er} / 1.6$ & $\mathrm{Tm} / 7.2$ & $\mathrm{Tm} / 1.6$ \\
\hline$a(\AA)$ & $7.3517(7)$ & $7.3459(7)$ & $7.3327(9)$ & 7.328 (1) & $7.320(3)$ & $7.300(2)$ & $7.2930(9)$ & $7.285(1)$ \\
\hline$x_{R}$ & $0.1756(8)$ & 0.175 (1) & $0.1758(8)$ & $0.176(1)$ & $0.173(2)$ & $0.174(2)$ & $0.1741(8)$ & $0.174(1)$ \\
\hline$x_{\mathrm{Ni}}$ & $0.3788(6)$ & $0.3785(9)$ & $0.3794(6)$ & $0.3791(8)$ & 0.380 (1) & 0.380 (1) & $0.3799(6)$ & $0.3798(8)$ \\
\hline NN (§) & $3.65(1)$ & $3.63(1)$ & $3.6084(5)$ & $3.6078(6)$ & $3.58(2)$ & $3.59(2)$ & $3.5770(5)$ & $3.5724(6)$ \\
\hline$R_{f}$ & 0.0310 & 0.0177 & 0.0325 & 0.0299 & 0.0590 & 0.0413 & 0.0428 & 0.0261 \\
\hline
\end{tabular}

A typical neutron powder diffraction pattern in the paramagnetic state (taken at $T=7.2 \mathrm{~K}$ for $\mathrm{Tm}_{2} \mathrm{Ni}_{1.78} \mathrm{In}$ ) is shown in Fig. 1. Similar patterns are observed for the other investigated samples. The tetragonal crystal structure of the $\mathrm{Mo}_{2} \mathrm{Fe} \mathrm{B}_{2}$ type (space group $P 4 / \mathrm{mbm}$ ) is shown in Fig. 2. The determined unit-cell parameters and atomic positional parameters are listed in Table 2. They are in good agreement with the previously reported data (Kalychak et al., 1990; Tyvanchuk et al., 2012).

\subsection{Magnetic properties}

Magnetic measurements were performed for the $R_{2} \mathrm{Ni}_{1.78} \mathrm{In}$ ( $R=\mathrm{Ho}$, Er and $\mathrm{Tm}$ ) samples. The data for $R=$ Ho and Er are in agreement with those reported previously (Tyvanchuk et al., 2012) and so they are not presented in this work. The results of magnetic measurements for $\mathrm{Tm}_{2} \mathrm{Ni}_{1.78} \mathrm{In}$ are presented in Fig. 3. At high temperatures the reciprocal magnetic susceptibility obeys the Curie-Weiss law with a negative value of the paramagnetic Curie temperature $(-9.8 \mathrm{~K})$ and the effective magnetic moment per Tm atom $\left(7.77 \mu_{\mathrm{B}}\right)$ being close to that predicted for the free $\mathrm{Tm}^{3+}$ ion $\left(7.56 \mu_{\mathrm{B}}\right)$. In the low-

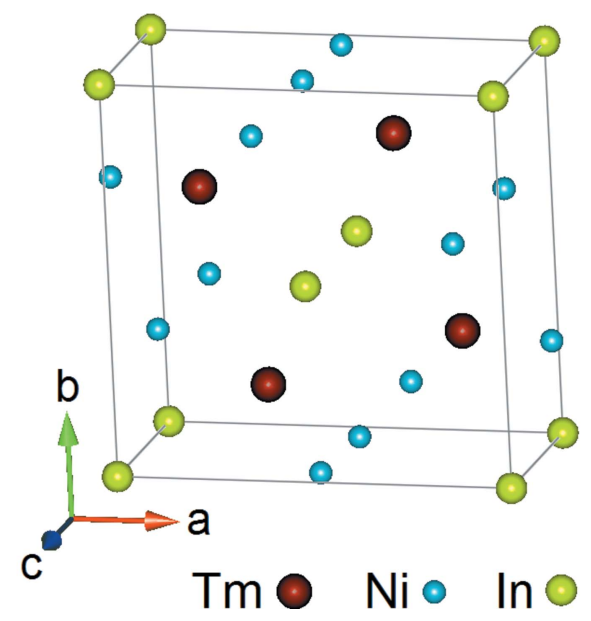

Figure 2

The crystal unit cell of $\mathrm{Tm}_{2} \mathrm{Ni}_{1.78} \mathrm{In}$, typical of other $R_{2} \mathrm{Ni}_{1.78} \mathrm{In}$ compounds (tetragonal crystal structure of the $\mathrm{Mo}_{2} \mathrm{FeB}_{2}$ type, space group $\mathrm{P} 4 / \mathrm{mbm}$ ). temperature range (see the upper inset in Fig. 3) a maximum typical of an antiferro- to paramagnetic transition is clearly visible at $4.5 \mathrm{~K}$. The lower inset in Fig. 3 presents the hysteresis loop of magnetization versus external magnetic field up to $90 \mathrm{kOe}$. The data were taken at $2.0 \mathrm{~K}$. It is worth noting that the value of the magnetic moment per Tm atom, as derived from the measurements at $T=2.0 \mathrm{~K}$ and $H=90 \mathrm{kOe}$, equals $3.4 \mu_{\mathrm{B}}$ and is significantly smaller than that of the free $\mathrm{Tm}^{3+}$ ion, which equals $7.0 \mu_{\mathrm{B}}$. The initial magnetization curve shows a metamagnetic transition at the critical field of $13.7 \mathrm{kOe}$.

\subsection{Magnetic structures}

The magnetic moments in $R_{2} \mathrm{Ni}_{1.78}$ In $(R=\mathrm{Tb}, \mathrm{Ho}, \mathrm{Er}, \mathrm{Tm})$ are expected to be localized on the rare earth atoms, as the effective magnetic moments per rare earth atom $R$ are close to the values predicted for the free $R^{3+}$ ions [see Section 3.2, as well as the data in Table II of Tyvanchuk et al. (2012)]. The

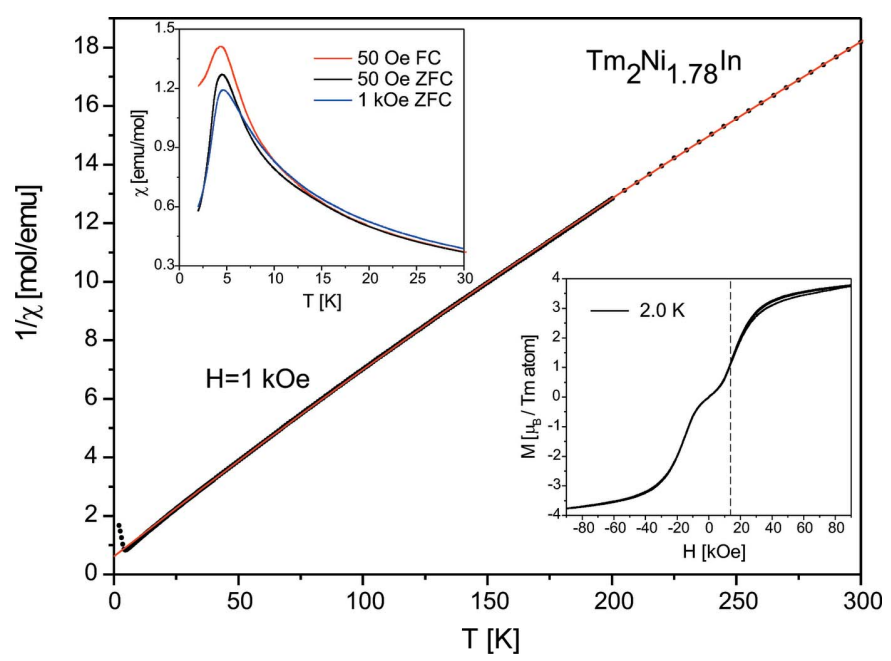

Figure 3

The reciprocal magnetic susceptibility for $\mathrm{Tm}_{2} \mathrm{Ni}_{1.78} \mathrm{In}$ (circles), together with a fitted line representing the Curie-Weiss law. The upper inset shows the low-temperature behaviour, while the lower one shows the hysteresis loop of the magnetization versus external magnetic field up to $90 \mathrm{kOe}$. The vertical line indicates the metamagnetic transition. 
rare earths in $R_{2} \mathrm{Ni}_{1.78}$ In occupy the $4 h$ Wyckoff site in the crystal unit cell (space group P4/mbm, see Fig. 2) (Szytuła et al., 2013). The coordinates of the four $R$ atoms in the unit cell are as follows:

(i) $R 1$ at $\left(x_{R}, x_{R}+\frac{1}{2}, \frac{1}{2}\right)$.

(ii) $R 2$ at $\left(x_{R}+\frac{1}{2}, 1-x_{R}, \frac{1}{2}\right)$.

(iii) $R 3$ at $\left(\frac{1}{2}-x_{R}, x_{R}, \frac{1}{2}\right)$.

(iv) $R 4$ at $\left(1-x_{R}, \frac{1}{2}-x_{R}, \frac{1}{2}\right)$.

Propagation vectors describing the magnetic ordering were obtained based on the positions of magnetic reflections in the neutron diffraction patterns of $R_{2} \mathrm{Ni}_{1.78} \mathrm{In}$. Subsequently, the allowed magnetic structures were calculated using the $B A S I R E P S$ program from the FULLPROF package. The program generates possible irreducible representations (IRs) for a given propagation vector, space group and Wyckoff position of magnetic atoms. The proper IRs, describing the magnetic structure for a selected $R_{2} \mathrm{Ni}_{1.78}$ In compound, were chosen as those giving the best agreement between the experimental and calculated patterns (the full symmetry analysis is available in the supporting information). The final magnetic structures in $R_{2} \mathrm{Ni}_{1.78} \mathrm{In}(R=\mathrm{Tb}, \mathrm{Ho}, \mathrm{Tm})$ were obtained by Rietveld refinement applied to the difference patterns (i.e. those obtained by subtraction of the neutron diffraction pattern in the paramagnetic state from the low- temperature pattern) as containing purely the magnetic contribution. The only exception was made for $R=\mathrm{Er}$, where the full low-temperature neutron diffraction pattern was used for Rietveld refinement of the magnetic structure because of the high noise in the difference pattern.

3.3.1. $\mathrm{Tb}_{2} \mathrm{Ni}_{1.78} \mathrm{In}$. The low-temperature magnetic structure has been reported by Szytuła et al. (2013). What is missing from the original paper is a symmetry analysis of the magnetic structure. Thus, a detailed symmetry analysis with a new refinement of the neutron powder diffraction data is presented below. A comparison with the previously reported data is presented at the end of this section.

The magnetic reflections in the neutron diffraction pattern registered at $1.6 \mathrm{~K}$ can be indexed by the propagation vector $\mathbf{k}_{1}=\left[\frac{1}{4}, \frac{1}{4}, \frac{1}{2}\right]$, as shown for the difference pattern $1.6 \mathrm{~K}-29.9 \mathrm{~K}$ in Fig. 4(a). The large magnetic unit cell consists of 32 crystal unit cells. It is enlarged four times along the $a$ and $b$ directions and twice along the $c$ direction. For the $\mathbf{k}_{1}=\left[\frac{1}{4}, \frac{1}{4}, \frac{1}{2}\right]$ propagation vector and the $4 h$ site of the $P 4 / \mathrm{mbm}$ space group, the $\mathrm{Tb}$ atoms are divided into three independent orbits: atom $\mathrm{Tb} 1$ in orbit I, a (Tb2, Tb3) pair in orbit II and atom Tb4 in orbit III. Therefore the magnetic ordering of the ( $\mathrm{Tb} 2, \mathrm{~Tb} 3)$ atoms is constrained by symmetry, while the magnetic moments of $\mathrm{Tb} 1$ and $\mathrm{Tb} 4$ are independent.
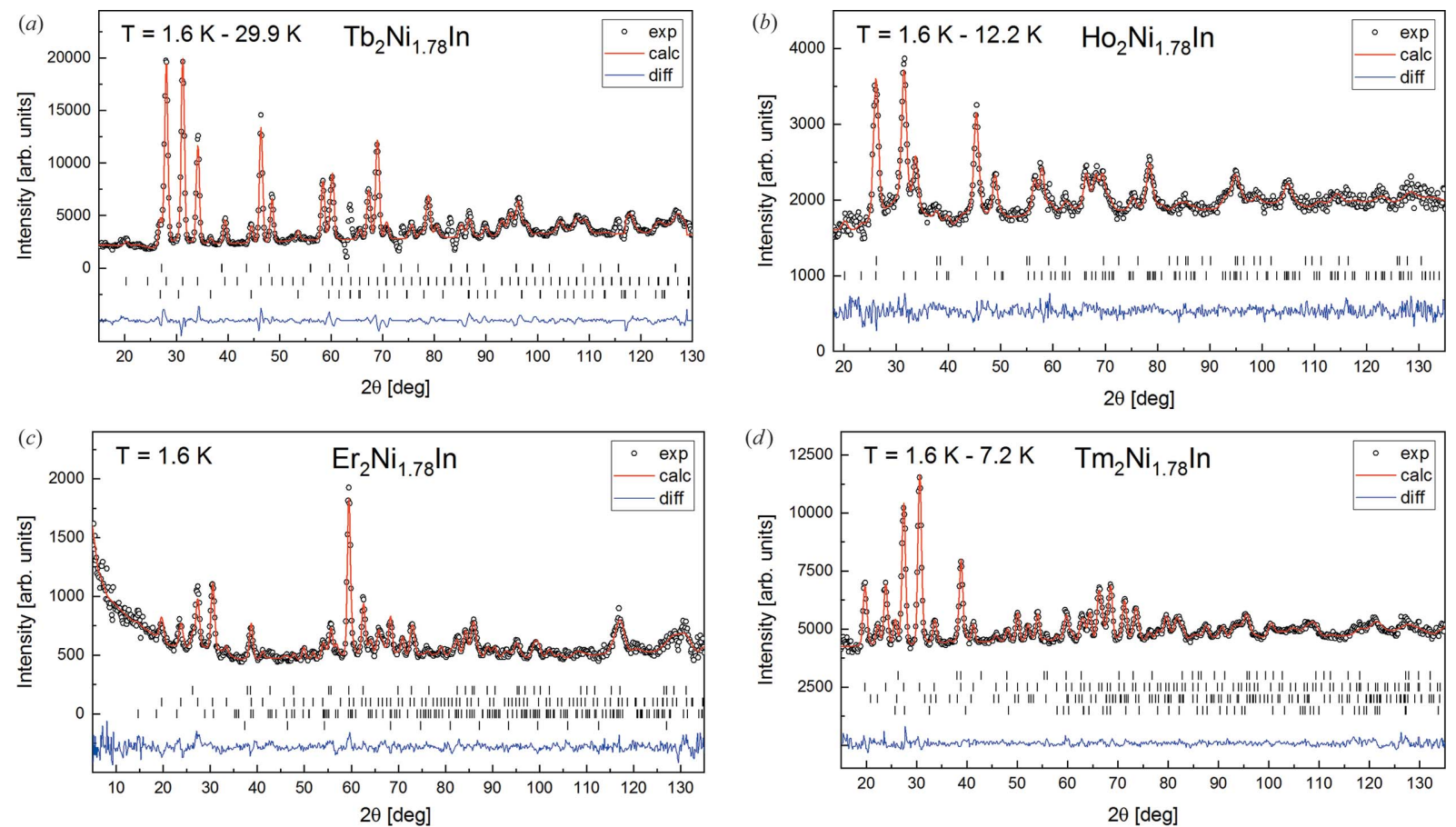

Figure 4

Neutron diffraction patterns for $R_{2} \mathrm{Ni}_{1.78}$ In collected at $T=1.6 \mathrm{~K}$ for $R=(a) \mathrm{Tb},(b) \mathrm{Ho},(c)$ Er and $(d) \mathrm{Tm}$ (open circles), with the results of the Rietveld refinements (red lines) and the difference curves (bottom blue lines). In panels $(a),(b)$ and $(d)$ the difference patterns are obtained by subtraction of the pattern collected in the paramagnetic state from the pattern collected in the magnetically ordered state at $T=1.6 \mathrm{~K}$. The first and second rows of vertical bars refer to the positions of reflections of nuclear and magnetic origin, respectively (the positions of nuclear reflections in the difference patterns are shown for reference only). The propagation vectors describing the magnetic structure are as follows: $(a) \mathbf{k}_{1}=\left[\frac{1}{4}, \frac{1}{4}, \frac{1}{2}\right]$ and $(b) \mathbf{k}_{2}=\left[0.31,0.31, \frac{1}{2}\right]$, while in $(c)$ and $(d)$ two propagation vectors $\left(\mathbf{k}_{1}=\left[\frac{1}{4}, \frac{1}{4}, \frac{1}{2}\right]\right.$ and $\left.\mathbf{k}_{1}^{\prime \prime \prime}=\left[-\frac{1}{4}, \frac{1}{4},-\frac{1}{2}\right]\right)$ are taken into consideration. In addition, the third row in $(a)$ refers to magnetic reflections arising from magnetic ordering in the $\mathrm{Tb}_{2} \mathrm{Ni}_{2} \mathrm{In}$ impurity phase (Szytuła et al., 2013). The third and fourth rows in (c) indicate nuclear reflections related to the $\mathrm{Er}_{5} \mathrm{Ni}_{2} \mathrm{In}_{4}$ (Gondek et al., 2012) [16.8 (2.3) wt\%] and $\mathrm{Er}_{2} \mathrm{O}_{3}$ (Maslen et al., 1996) [6.3 (5) wt\%] impurities. The third and fourth rows in $(d)$ refer to the magnetic ordering in $\mathrm{Tm}_{5} \mathrm{Ni}_{2} \mathrm{In}_{4}$ (Szytuła et al., 2014) [10.8 (1.4) wt \%] and $\mathrm{Tm}_{2} \mathrm{Ni}_{2} \mathrm{In}$ (Baran et al., 2017) [6.6 (8) wt\%]. Selected $2 \theta$ regions in $(a)$ have been excluded from the refinement as they contain anomalies arising from shifts in nuclear reflections due to thermal evolution of the unit-cell parameters. 
Table 3

The magnetic structure of $\mathrm{Tb}_{2} \mathrm{Ni}_{1.78}$ In described by the IR2 representation for the $4 h$ Wyckoff site of the $P 4 / \mathrm{mbm}$ space group and propagation vector $\mathbf{k}_{1}=\left[\frac{1}{4}, \frac{1}{4}, \frac{1}{2}\right]$.

$C_{1}$ and $\Phi$ are, respectively, an amplitude and a phase factor of modulation of the magnetic moment along the BV1 vector, $\mu$ is the magnetic moment of the rare earth atom, and $R_{\text {magn }}$ is the magnetic reliability factor of the Rietveld refinement.

\begin{tabular}{|c|c|c|}
\hline & $\mathbf{k}_{1}=\left[\frac{1}{4}, \frac{1}{4}, \frac{1}{2}\right]$ & BV1 \\
\hline $4 h$ & $\begin{array}{l}\operatorname{Tb} 1\left(x_{\mathrm{Tb}}, x_{\mathrm{Tb}}+\frac{1}{2}, \frac{1}{2}\right) \\
\operatorname{Tb} 2\left(x_{\mathrm{Tb}}+\frac{1}{2}, 1-x_{\mathrm{Tb}}, \frac{1}{2}\right) \\
\operatorname{Tb} 3\left(\frac{1}{2}-x_{\mathrm{Tb}}, x_{\mathrm{Tb}}, \frac{1}{2}\right) \\
\operatorname{Tb} 4\left(1-x_{\mathrm{Tb}}, \frac{1}{2}-x_{\mathrm{Tb}}, \frac{1}{2}\right)\end{array}$ & $\begin{array}{lll}0 & 0 & 1 \\
0 & 0 & -i \\
0 & 0 & 1 \\
0 & 0 & 1\end{array}$ \\
\hline
\end{tabular}

\begin{tabular}{llrlll}
\hline$T(\mathrm{~K})$ & $\mathrm{Tb}_{2} \mathrm{Ni}_{1.78} \mathrm{In}$ & \multicolumn{1}{c}{$C_{1}\left(\mu_{\mathrm{B}}\right)$} & $\Phi(2 \pi)$ & $\mu\left(\mu_{\mathrm{B}}\right)$ & $R_{\text {magn }}$ \\
\hline 1.6 & $\mathrm{~Tb} 1$ & $10.67(7)$ & 0.125 & $7.55(5)$ & 0.0542 \\
& $\mathrm{~Tb} 2$ & $10.67(7)$ & 0.625 & $7.55(5)$ & \\
& $\mathrm{Tb} 3$ & $10.67(7)$ & 0.375 & $7.55(5)$ & \\
& $\mathrm{Tb} 4$ & $-10.67(7)$ & 0.375 & $7.55(5)$ & \\
\hline
\end{tabular}

Theory predicts four irreducible representations, namely IR1 and IR3, related to the ordering of magnetic moments within the $a b$ plane, and IR2 and IR4, related to the ordering along the $c$ axis. The best agreement with the experimental pattern [Fig. 4(a)] is obtained for an antiferromagnetic ordering along the $c$ direction, described by the IR2 representation (Table 3). Although the symmetry analysis allows for different amplitudes of modulation for the magnetic moments of atoms belonging to different orbits, it was reasonable to assume an identical absolute value of the $C_{1}$ parameter (see Table 3) for all $\mathrm{Tb}$ atoms.

As only the relative phase shifts between atoms influence the intensities of the magnetic peaks, the absolute value of the magnetic phase factor of the $\mathrm{Tb} 1$ reference atom can be chosen arbitrarily, taking into account the physical validity of the proposed magnetic structure. For the zero phase factor of
Table 4

The magnetic structure of $\mathrm{Ho}_{2} \mathrm{Ni}_{1.78}$ In described by the IR2 representation for the $4 h$ Wyckoff site of the $P 4 / \mathrm{mbm}$ space group and propagation vector $\mathbf{k}_{2}=\left[k_{x}, k_{x}, \frac{1}{2}\right]$, where $k_{x}=0.3074(4)$ at $1.6 \mathrm{~K}$.

$C_{1}$ and $\Phi$ are, respectively, an amplitude and a phase factor of modulation of the magnetic moment along the BV1 vector, $\mu_{\max }$ is the maximum magnetic moment of the rare earth atom and $R_{\text {magn }}$ is the magnetic reliability factor of the Rietveld refinement. The parameter $\alpha$ is defined as $\alpha=2 \pi k_{x}$.

\begin{tabular}{|c|c|c|c|c|c|}
\hline & \multicolumn{3}{|c|}{$\mathbf{k}_{2}=\left[0.31,0.31, \frac{1}{2}\right]$} & \multicolumn{2}{|l|}{ BV1 } \\
\hline $4 h$ & \multicolumn{3}{|c|}{$\begin{array}{l}\text { Ho1 }\left(x_{\mathrm{Ho}}, x_{\mathrm{Ho}}+\frac{1}{2}, \frac{1}{2}\right) \\
\text { Ho2 }\left(x_{\mathrm{Ho}}+\frac{1}{2}, 1-x_{\mathrm{Ho}}, \frac{1}{2}\right) \\
\text { Ho3 }\left(\frac{1}{2}-x_{\mathrm{Ho}}, x_{\mathrm{Ho}}, \frac{1}{2}\right) \\
\text { Ho4 }\left(1-x_{\mathrm{Ho}}, \frac{1}{2}-x_{\mathrm{Ho}}, \frac{1}{2}\right)\end{array}$} & \multicolumn{2}{|c|}{$\begin{array}{lll}0 & 0 & 1 \\
0 & 0 & (\cos \alpha-i \sin \alpha) \\
0 & 0 & 1 \\
0 & 0 & 1\end{array}$} \\
\hline$T(\mathrm{~K})$ & $\mathrm{Ho}_{2} \mathrm{Ni}_{1.78} \mathrm{In}$ & $C_{1}\left(\mu_{\mathrm{B}}\right)$ & $\Phi(2 \pi)$ & $\mu_{\max }\left(\mu_{\mathrm{B}}\right)$ & $R_{\text {magn }}$ \\
\hline 1.6 & $\begin{array}{l}\text { Ho1 } \\
\text { Ho2 } \\
\text { Ho3 } \\
\text { Ho4 }\end{array}$ & $\begin{array}{l}7.93(8) \\
7.93(8) \\
7.93(8) \\
7.93(8)\end{array}$ & $\begin{array}{l}0 \dagger \\
0.55(2) \\
0.24(2) \\
-0.297(7)\end{array}$ & $\begin{array}{l}7.93(8) \\
7.93(8) \\
7.93(8) \\
7.93(8)\end{array}$ & 0.0552 \\
\hline
\end{tabular}

$\dagger$ Fixed to define a point of reference.

atom $\mathrm{Tb} 1$ and the magnetic phase factors of $\mathrm{Tb} 2, \mathrm{~Tb} 3, \mathrm{~Tb} 4$ fixed to $\frac{1}{2}, \frac{1}{4}, \frac{1}{4}$ (in $2 \pi$ units), respectively, the magnetic moment on a particular $\mathrm{Tb}$ atom, as determined for $T=1.6 \mathrm{~K}$, is equal either to zero or to the modulation amplitude $\left|C_{1}\right|=$ 10.67 (7) $\mu_{\mathrm{B}}$.

A more realistic magnetic structure [see Fig. 5(a)] is obtained while assuming a magnetic phase factor of atoms $\mathrm{Tb} 1$ of $\frac{1}{8}$, with the phase factors of atoms $\mathrm{Tb} 2, \mathrm{~Tb} 3, \mathrm{~Tb} 4$ fixed to $\frac{5}{8}, \frac{3}{8}$ and $\frac{3}{8}$, respectively. For the latter magnetic structure model, all terbium magnetic moments equal $\mu=\left|C_{1}\right| /\left(2^{1 / 2}\right)=7.55$ (5) $\mu_{\mathrm{B}}$ at $T=1.6 \mathrm{~K}$.

The magnetic structure presented above for $\mathrm{Tb}_{2} \mathrm{Ni}_{1.78} \mathrm{In}$ coincides with the structure reported originally by Szytuła $e t$ al. (2013), although the new refinement leads to a better value of the reliability factor $\left(R_{\mathrm{magn}}=0.054\right)$ than the one originally reported (0.081).

\section{$\mathrm{Tb}_{2} \mathrm{Ni}_{1.78} \mathrm{In}$}

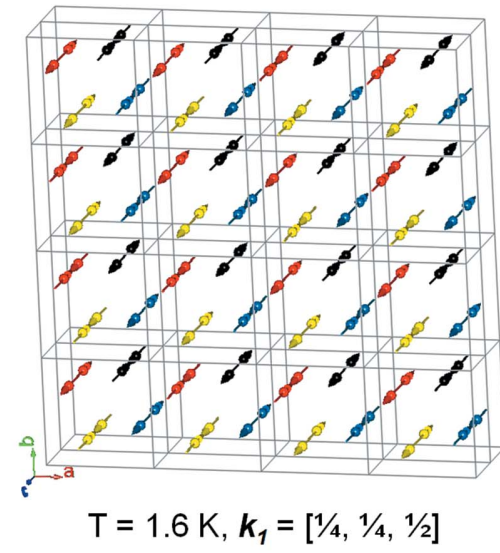

(a)

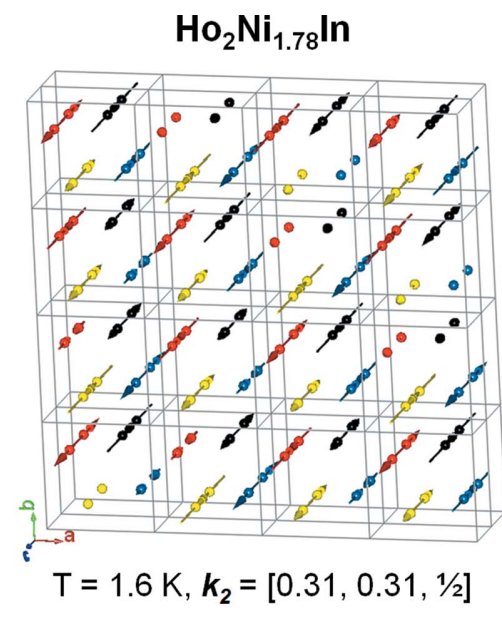

(b)

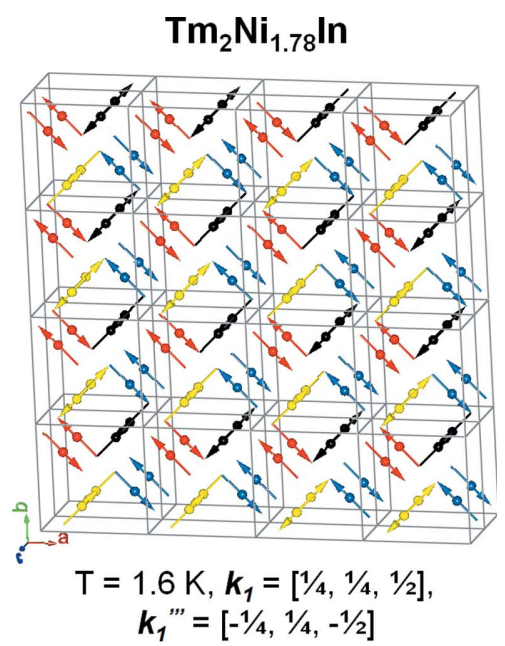

(c)

Figure 5

Magnetic structures in $R_{2} \mathrm{Ni}_{1.78} \mathrm{In}$ as described in Tables 3, 4 and 5: (a) $R=\mathrm{Tb},(b) R=$ Ho and $(c) R=\mathrm{Er}$, Tm (presented here for $R=\mathrm{Tm}$ ). The magnetic unit cell is shown for commensurate structures in panels $(a)$ and $(c)$, while for the incommensurate structure in $(b)$ a representative fragment of the structure is shown. Atoms $R_{1}, R_{2}, R_{3}$ and $R_{4}$ are depicted in red, black, yellow and blue, respectively. 
Table 5

The magnetic structure of $R_{2} \mathrm{Ni}_{1.78} \operatorname{In}(R=\mathrm{Er}, \mathrm{Tm})$ described by the pair of propagation vectors $\mathbf{k}_{1}=\left[\frac{1}{4}, \frac{1}{4}, \frac{1}{2}\right]($ atoms $R 1$ and $R 4)$ and $\mathbf{k}_{1}^{\prime \prime \prime}=\left[-\frac{1}{4}, \frac{1}{4},-\frac{1}{2}\right]($ atoms $R 2$ and $R 3$ ).

The ordering of magnetic moments is related to the IR1 representation for each propagation vector. The $C_{i}$ coefficients describe individual contributions to the rare earth magnetic moment $\mu$ along the respective basis vectors, while $\Phi$ is the magnetic phase factor. $R_{\text {magn }}$ denotes the magnetic reliability factor of the Rietveld refinement

\begin{tabular}{|c|c|c|c|c|c|c|c|c|}
\hline & & \multicolumn{2}{|c|}{$\mathbf{k}_{1}=\left[\frac{1}{4}, \frac{1}{4}, \frac{1}{2}\right]$} & \multicolumn{2}{|c|}{$\mathbf{k}_{1}^{\prime \prime \prime}=\left[-\frac{1}{4}, \frac{1}{4},-\frac{1}{2}\right]$} & & & \\
\hline & & BV1 & BV2 & BV1 & BV2 & & & \\
\hline $4 h$ & $\begin{array}{l}R 1\left(x_{R}, x_{R}+\frac{1}{2}, \frac{1}{2}\right) \\
R 2\left(x_{R}+\frac{1}{2}, 1-x_{R}, \frac{1}{2}\right) \\
R 3\left(\frac{1}{2}-x_{R}, x_{R}, \frac{1}{2}\right) \\
R 4\left(1-x_{R}, \frac{1}{2}-x_{R}, \frac{1}{2}\right)\end{array}$ & $\begin{array}{lll}1 & -1 & 0 \\
0 & i & 0 \\
1 & 0 & 0 \\
1 & -1 & 0\end{array}$ & $\begin{array}{lll}i & 0 & 0 \\
0 & 1 & 0\end{array}$ & $\begin{array}{lll}1 & 0 & 0 \\
1 & 1 & 0 \\
1 & 1 & 0 \\
0 & i & 0\end{array}$ & 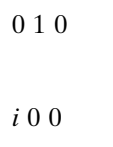 & & & \\
\hline$T(\mathrm{~K})$ & $\mathrm{Er}_{2} \mathrm{Ni}_{1.78} \mathrm{In}$ & $C_{1}\left(\mu_{\mathrm{B}}\right)$ & $C_{2}\left(\mu_{\mathrm{B}}\right)$ & $C_{1}\left(\mu_{\mathrm{B}}\right)$ & $C_{2}\left(\mu_{\mathrm{B}}\right)$ & $\Phi(2 \pi)$ & $\mu\left(\mu_{\mathrm{B}}\right)$ & $R_{\text {magn }}$ \\
\hline$T(\mathrm{~K})$ & $\mathrm{Tm}_{2} \mathrm{Ni}_{1.78} \mathrm{In}$ & $C_{1}\left(\mu_{\mathrm{B}}\right)$ & $C_{2}\left(\mu_{\mathrm{B}}\right)$ & $C_{1}\left(\mu_{\mathrm{B}}\right)$ & $C_{2}\left(\mu_{\mathrm{B}}\right)$ & $\Phi(2 \pi)$ & $\mu\left(\mu_{\mathrm{B}}\right)$ & $R_{\text {magn }}$ \\
\hline 3.6 & $\begin{array}{l}\text { Tm1 } \\
\text { Tm2 } \\
\text { Tm3 } \\
\text { Tm4 }\end{array}$ & $\begin{array}{r}3.73(7) \\
-3.73(7)\end{array}$ & & $\begin{array}{r}-3.73(7) \\
3.73(7)\end{array}$ & & $\begin{array}{l}0.125 \\
0.375 \\
0.125 \\
0.375\end{array}$ & $\begin{array}{l}3.73(7) \\
3.73(7) \\
3.73(7) \\
3.73(7)\end{array}$ & 0.121 \\
\hline 1.6 & $\begin{array}{l}\text { Tm1 } \\
\text { Tm2 } \\
\text { Tm3 } \\
\text { Tm4 }\end{array}$ & $-6.09(4)$ & & $\begin{array}{r}-6.09(4) \\
6.09(4)\end{array}$ & & $\begin{array}{l}0.125 \\
0.375 \\
0.125 \\
0.375\end{array}$ & $\begin{array}{l}6.09(4) \\
6.09(4) \\
6.09(4) \\
6.09(4)\end{array}$ & 0.0476 \\
\hline
\end{tabular}

3.3.2. $\mathrm{Ho}_{2} \mathrm{Ni}_{1.78} \mathrm{In}$. The magnetic contribution to the $\mathrm{Ho}_{2} \mathrm{Ni}_{1.78} \mathrm{In}$ diffraction pattern at $T=1.6 \mathrm{~K}$ is shown in Fig. 4(b). The propagation vector indexing the magnetic reflections observed for $\mathrm{Ho}_{2} \mathrm{Ni}_{1.78} \mathrm{In}$ is an incommensurate one, $\mathbf{k}_{2}=\left[k_{x}, k_{x}, \frac{1}{2}\right]$, where $k_{x}=0.3074$ (4).

The symmetry analysis shows that the Ho atoms are divided into three orbits, namely, Ho1 in orbit I, the (Ho2, Ho3) pair in orbit II and Ho4 in orbit III, and that the ordering of magnetic moments is allowed both within the $a b$ plane (IR1, IR3) and along the $c$ direction (IR2, IR4). The agreement with the experimental difference pattern $1.6 \mathrm{~K}-12.2 \mathrm{~K}$ [Fig. $4(b)]$ is obtained for the IR2 irreducible representation, i.e. the ordering of magnetic moments along the $c$ axis (see Table 4).

The amplitude of modulation of the magnetic moments, constrained to be identical for all Ho atoms, is equal to $C_{1}=$ 7.93 (8) $\mu_{\mathrm{B}}$. As for an incommensurate magnetic structure, the absolute values of the magnetic phase factors $\Phi_{i}$ have no physical meaning. Thus, the phase factor of Ho1 was fixed to zero in order to define a point of reference, while the phase factors of all remaining atoms were refined. The magnetic phase factors for $\mathrm{Ho} 3$ and $\mathrm{Ho} 4$ have opposite signs, but absolute values close to $\frac{1}{4}$ (while assuming $\Phi_{1}=0$ ), and for Ho2 the phase factor is $\Phi_{3}+k_{x} \simeq \frac{1}{2}$. A fragment of the antiferromagnetic structure of $\mathrm{Ho}_{2} \mathrm{Ni}_{1.78} \mathrm{In}$ (a magnetic unit cell cannot be defined due to incommensurability) is presented in Fig. 5(b).

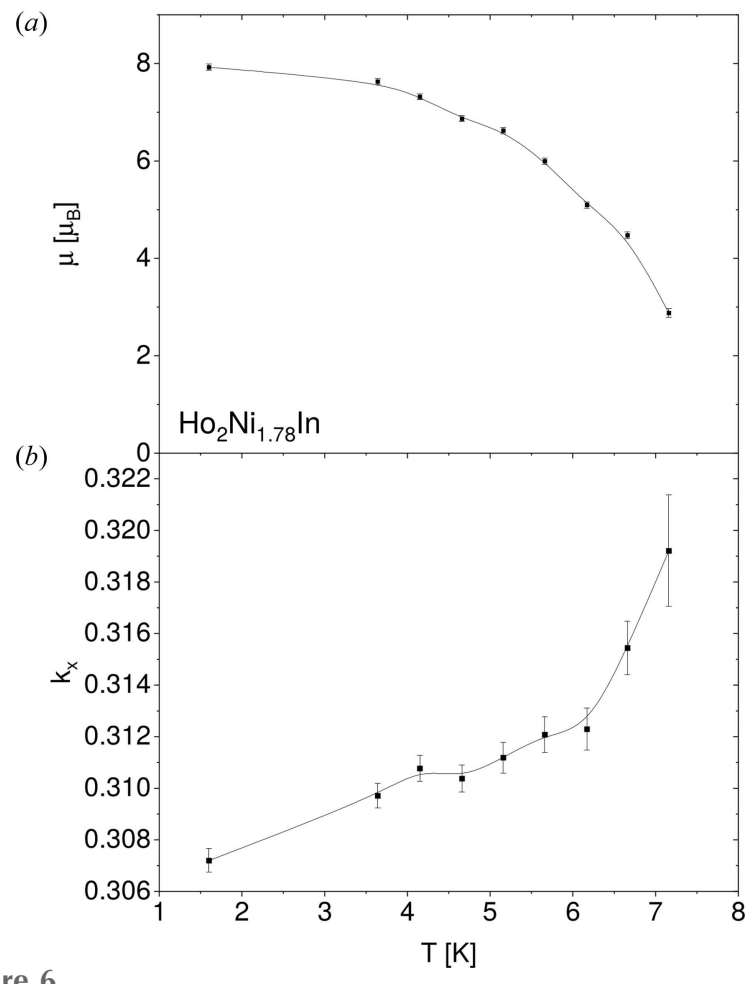

Figure 6

$\mathrm{Ho}_{2} \mathrm{Ni}_{1.78} \mathrm{In}$ : temperature dependence of $(a)$ the amplitude of modulation of the magnetic moment $\left(C_{1}\right.$ parameter $)$ and $(b)$ the $k_{x}$ component of the propagation vector $\mathbf{k}_{2}=\left[k_{x}, k_{x}, \frac{1}{2}\right]$. 
In the case of $\mathrm{Ho}_{2} \mathrm{Ni}_{1.78} \mathrm{In}$, the thermal evolution of its neutron diffraction pattern was also recorded. Based on these data, the temperature dependences of the amplitude of modulation ( $C_{1}$ parameter) and the $k_{x}$ component of the propagation vector were determined and are presented in Fig. 6. The temperature dependence of $C_{1}$ yields $T_{\mathrm{N}}=7.5 \mathrm{~K}$. It is also worth noting that the $k_{x}$ component of the propagation vector increases noticeably while approaching $T_{\mathrm{N}}$.

3.3.3. $\mathrm{Er}_{2} \mathrm{Ni}_{1.78} \mathrm{In}$ and $\mathrm{Tm}_{2} \mathrm{Ni}_{1.78} \mathrm{In}$. The magnetic contributions to the neutron patterns of $\mathrm{Er}_{2} \mathrm{Ni}_{1.78} \mathrm{In}$ and $\mathrm{Tm}_{2} \mathrm{Ni}_{1.78} \mathrm{In}$ are similar to one another, indicating the same magnetic structure in both compounds, with the only difference being in the absolute values of the magnetic moments. The magnetic reflections observed at $T=1.6 \mathrm{~K}$ for $\mathrm{Er}_{2} \mathrm{Ni}_{1.78} \mathrm{In}$ [Fig. 4(c)] and $\mathrm{Tm}_{2} \mathrm{Ni}_{1.78}$ In [Fig. $4(d)$ ] can be indexed by the propagation vector $\mathbf{k}_{1}=\left[\frac{1}{4}, \frac{1}{4}, \frac{1}{2}\right]$.

In contrast to what was found for $R=\mathrm{Tb}$, agreement with the experimental patterns for $R=\mathrm{Er}$, Tm was obtained for the magnetic moments constrained to the $a b$ plane according to the IR1 representation. However, this initial model of the magnetic structure in $R_{2} \mathrm{Ni}_{1.78} \mathrm{In}(R=\mathrm{Er}$, Tm) leads to unreliable values of the magnetic moments, i.e. following the results of Rietveld refinement, the moments on atoms $R 1$ and $R 4$ equal 9.2 (2) and 8.62 (5) $\mu_{\mathrm{B}}$ for $R=$ Er and Tm, respectively, while atoms $R 2$ and $R 3$ do not contribute at all to the intensity of the magnetic peaks as they have zero magnetic moments. This non-physical result points to the necessity of taking into consideration additional propagation vectors,

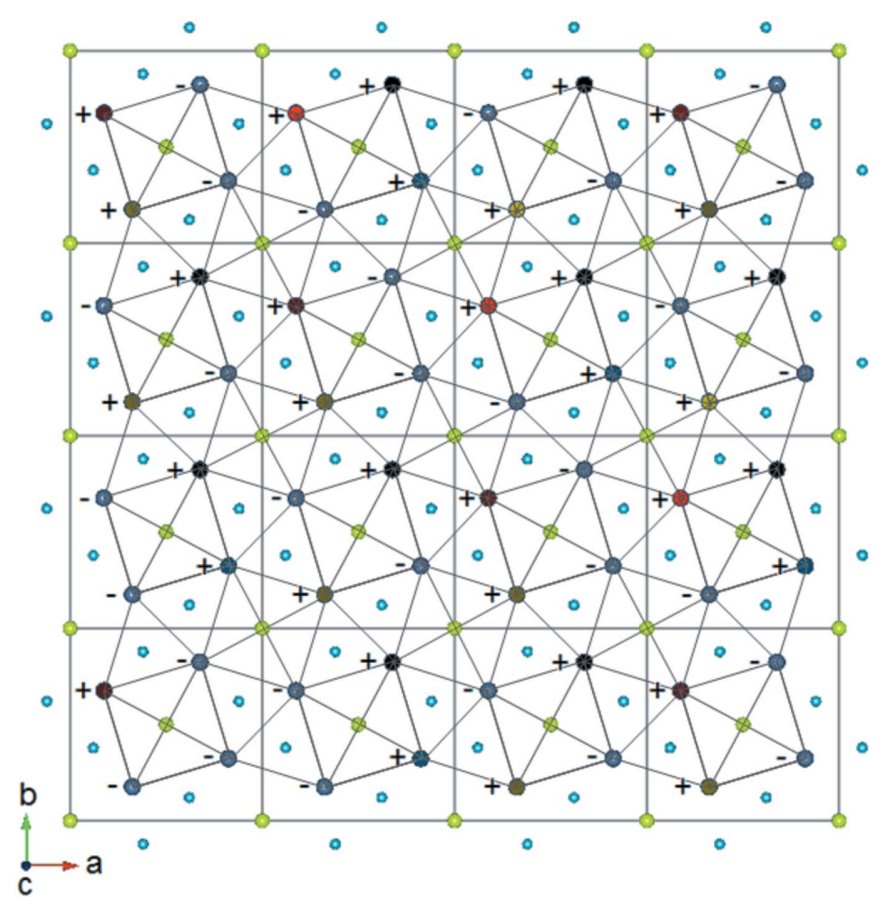

Figure 7

A projection of the crystal and magnetic structures in $\mathrm{Ho}_{2} \mathrm{Ni}_{1.78} \mathrm{In}$ on the (001) plane. The + and - symbols indicate the parallel and antiparallel orientations, respectively, of the Ho magnetic moments with respect to the $c$ axis. Triangles and squares formed by the rare earth atoms are also marked. similar to what was previously reported for TmAgGe (Baran et al., 2009).

The $\mathbf{k}_{1}=\left[\frac{1}{4}, \frac{1}{4}, \frac{1}{2}\right]$ vector forms a star containing three other propagation vectors, namely, $\mathbf{k}_{1}^{\prime}=\left[-\frac{1}{4},-\frac{1}{4}, \frac{1}{2}\right], \mathbf{k}_{1}^{\prime \prime}=\left[\frac{1}{4},-\frac{1}{4}, \frac{1}{2}\right]$ and $\mathbf{k}_{1}^{\prime \prime \prime}=\left[-\frac{1}{4}, \frac{1}{4},-\frac{1}{2}\right]$; all these propagation vectors provide the same positions of Bragg reflections of the magnetic origin. $\mathbf{k}_{1}^{\prime}$ is equivalent to $-\mathbf{k}_{1}$ while $\mathbf{k}_{1}^{\prime \prime \prime}$ is equivalent to $-\mathbf{k}_{1}^{\prime \prime}$. It is worth noting that taking a combination of the propagation vectors from the first $\left(\mathbf{k}_{1}, \mathbf{k}_{1}^{\prime}\right)$ and second $\left(\mathbf{k}_{1}^{\prime \prime}, \mathbf{k}_{1}^{\prime \prime \prime}\right)$ pairs leads to new magnetic structures that cannot be obtained with the use of the propagation vectors originating from a single pair. The Rietveld refinement shows that both good agreement with the experimental patterns and reasonable values of the magnetic moments can be obtained for a combination of the $\mathbf{k}_{1}$ and $\mathbf{k}_{1}^{\prime \prime \prime}$ vectors. The final structure is presented in Fig. 5(c).

The magnetic ordering of atoms $R 1$ and $R 4$ is described by $\mathbf{k}_{1}=\left[\frac{1}{4}, \frac{1}{4}, \frac{1}{2}\right]$, while that involving atoms $R 2$ and $R 3$ is related to $\mathbf{k}_{1}^{\prime \prime \prime}=\left[-\frac{1}{4}, \frac{1}{4},-\frac{1}{2}\right]$ (see Table 5). According to the symmetry analysis reported by the BASIREPS program, in the discussed structure the magnetic moments of $R 1, \ldots, R 4$ are independent with respect to the amplitude of modulation and the magnetic phase factor. However, in order to get a physically valid magnetic structure the amplitudes of modulation of all $R_{i}$ were constrained to be equal. The Rietveld refinement yields a difference of $\frac{1}{4}$ between the magnetic phase factors of atoms $R 2$ and $R 4$ and those of atoms $R 1$ and $R 3$. In order to obtain identical absolute values of the magnetic moments for all rare earth atoms, equal to $\left|C_{1}\right|$, the magnetic phases were fixed to $\frac{1}{8}$ for $R 1$ and $R 3$ and to $\frac{3}{8}$ for $R 2$ and $R 4$. The magnetic moments derived from refinement of the neutron diffraction patterns collected at $T=1.6 \mathrm{~K}$ equal 6.5 (1) $\mu_{\mathrm{B}}$ for $R=\mathrm{Er}$ and 6.09 (4) $\mu_{\mathrm{B}}$ for $R=\mathrm{Tm}$.

\section{Discussion}

The X-ray and neutron diffraction data confirm that the $R_{2} \mathrm{Ni}_{1.78} \mathrm{In}(R=\mathrm{Tb}, \mathrm{Ho}, \mathrm{Er}$ and $\mathrm{Tm})$ compounds crystallize in the tetragonal $\mathrm{Mo}_{2} \mathrm{FeB}_{2}$-type structure (space group $P 4 / \mathrm{mbm}$ ) in a broad temperature range down to $1.6 \mathrm{~K}$. In this structure, the rare earth atoms occupy the $4 h$ Wyckoff site in the $a b$ plane $\left(z=\frac{1}{2}\right)$ separated by planes containing the $\mathrm{Ni}$ and In atoms $(z=0)$. The structure is highly anisotropic with a short $c$ lattice parameter (the $c / a$ ratio is close to 0.5 ). The atomic arrangement is formed from slabs of CsCl-type ( $R \mathrm{In})$ and $\mathrm{AlB}_{2}$-type $\left(R \mathrm{Ni}_{2}\right)$. The rare earth atoms form triangles and squares within the $a b$ plane (see Fig. 7). The resultant natural anisotropic multilayer structure suggests a high anisotropy of physical properties, especially those of magnetic character.

Magnetic susceptibility measurements, taken in the paramagnetic state, show that the effective magnetic moments per rare earth atom are close to the free $R^{3+}$ ion values, and therefore suggest that only the rare earth atoms carry magnetic moments. This result is in agreement with the results of Rietveld refinement of the low-temperature neutron diffraction data. Therefore, the magnetism in the investigated compounds is related to the rare earth magnetic moments, 
while the $\mathrm{Ni}$ atoms either remain non-magnetic, as predicted by ab initio calculations of the electronic structure in $\mathrm{U}_{2} \mathrm{Ni}_{2} X$ ( $X=p$-electron element) (Diviš et al., 1995; Matar, 1995), or their magnetic moments are too small to be detected by the experimental methods used in this study, especially considering that the $\mathrm{Ni}$ atoms are accompanied by rare earth atoms carring large moments.

The paramagnetic Curie temperatures in $R_{2} \mathrm{Ni}_{1.78} \mathrm{In}$ are found to be negative, suggesting that antiferromagnetic interactions are predominant. The antiferromagnetic ordering in the investigated compounds is confirmed by both the lowtemperature magnetic data (see the upper inset in Fig. 3) and the neutron diffraction data (see Tables 3, 4 and 5). Detailed analysis of the low-temperature neutron diffraction data shows that the magnetic structures are related to the following propagation vectors: $\mathbf{k}_{1}=\left[\frac{1}{4}, \frac{1}{4}, \frac{1}{2}\right]$ for $R=\mathrm{Tb}$ and $\mathbf{k}_{2}=\left[k_{x}, k_{x}, \frac{1}{2}\right]$ for $R=\mathrm{Ho}$, where $k_{x}=0.3074$ (4) at $1.6 \mathrm{~K}$ and increases with increasing temperature, while for $R=\mathrm{Er}$ and Tm a combination of two propagation vectors $\left(\mathbf{k}_{1}=\left[\frac{1}{4}, \frac{1}{4}, \frac{1}{2}\right]\right.$ and $\mathbf{k}_{1}^{\prime \prime \prime}=\left[-\frac{1}{4}, \frac{1}{4}\right.$, $\left.\left.-\frac{1}{2}\right]\right)$ has to be taken into consideration. All these vectors have the $k_{z}$ component equal to $\frac{1}{2}$, indicating simple antiferromagnetic coupling between neighbouring (001) planes, while the ordering within the planes is much more complex [see Figs. 5(a)-5(c) and 7].

As the rare earth interatomic distances exceed $3.5 \AA$, they are high enough to exclude any direct interactions, and therefore the interactions between magnetic atoms are expected to be indirect of the Ruderman-Kittel-KasuyaYosida (RKKY) type. One of the predictions of the RKKY theory is proportionality between the critical temperature of magnetic ordering (i.e. the Curie or Néel temperature) and the de Gennes factor defined as $\left(g_{J}-1\right)^{2} J(J+1)$, where $g_{J}$ is the Lande splitting factor and $J$ is the total angular momentum of the corresponding magnetic ion (De Gennes, 1962). This relation is not fulfilled in $R_{2} \mathrm{Ni}_{1.78}$ In [see Fig. 4 of Tyvanchuk $e t$ al. (2012)], indicating an influence of the crystalline electric field (CEF) in the stabilization of the magnetic structure. The impact of the CEF also manifests itself in the reduction of the rare earth magnetic moment in the magnetically ordered state when compared with the moments of the free $R^{3+}$ ions - see Section 3.2 and the data in Table II of Tyvanchuk et al. (2012). A change in direction of the magnetic moment, from parallel to the $c$ axis in the Tb- and Ho-based compounds to perpendicular to this axis for $R=\mathrm{Er}$ and $\mathrm{Tm}$, is further proof of the important role of the CEF, as it coincides with a change in sign of the Stevens factor $\alpha_{J}$ from negative in $\mathrm{Tb}$ and Ho to positive in Er and Tm (Stevens, 1952).

Antiferromagnetic structures have also been observed in isostructural compounds with the $P 4 / \mathrm{mbm}$ space group. The propagation vector $\mathbf{k}=\left[0,0, \frac{1}{2}\right]$ describes the magnetic order in $\mathrm{Pr}_{2} \mathrm{Pd}_{2} \mathrm{In}$ (Fischer et al., 2000) and in the low-temperature magnetic phase in $\mathrm{Tb}_{2} \mathrm{Pd}_{2.05} \mathrm{Sn}_{0.95}$ (Laffargue et al., 1997), while the high-temperature magnetic phase in $\mathrm{Tb}_{2} \mathrm{Pd}_{2.05} \mathrm{Sn}_{0.95}$, which appears near the Néel temperature, is related to $\mathbf{k}=\left[k_{x}\right.$, $k_{x}, \frac{1}{2}$, where $k_{x}$ equals $0.075(5)$ at $T=20.8 \mathrm{~K}$ and 0.115 (5) at $26.3 \mathrm{~K}$. The magnetic order in $\mathrm{Nd}_{2} \mathrm{Pd}_{2} \mathrm{In}$ is described by the propagation vector $\mathbf{k}=\left[\frac{1}{4}, \frac{1}{4}, 0\right]$ (Fischer et al., 2000).
The observed variety of magnetic orderings within the (001) plane can be understood while taking into account the results of the theoretical analysis of the magnetic structure in the $\mathrm{TbCo}_{2} \mathrm{~B}_{2}$ compound with a tetragonal crystal structure (space group $P 4 / \mathrm{mmm}$ ). The magnetic order in $\mathrm{TbCo}_{2} \mathrm{~B}_{2}$ is described by the propagation vector $\mathbf{k}=\left[k_{x}, k_{x}, 0\right]$, where $k_{x}$ equals $\frac{1}{4}$ at $1.5 \mathrm{~K}$ and 0.244 at $14 \mathrm{~K}$ (André et al., 1991). The theoretical model considering interactions within the (001) plane between the first-, second- and third-nearest neighbours, described by the exchange integrals $J_{1}, J_{2}$ and $J_{3}$, respectively, shows that the magnetic phase related to $\mathbf{k}=\left[\frac{1}{4}, \frac{1}{4}, 0\right]$ is stabilized by a relatively large positive value of $J_{3}$ (Plumer \& Caillé, 1992).

\section{Conclusions}

The results presented in this work confirm that the ternary $R_{2} \mathrm{Ni}_{1.78} \mathrm{In}(R=\mathrm{Tb}, \mathrm{Ho}, \mathrm{Er}, \mathrm{Tm})$ compounds crystallize in the tetragonal $\mathrm{Mo}_{2} \mathrm{FeB}_{2}$-type structure (space group $\mathrm{P} 4 / \mathrm{mbm}$ ). The magnetic and neutron diffraction data indicate that the rare earth magnetic moments in these compounds order antiferromagnetically at low temperatures, with the magnetic structures described by the propagation vector $\mathbf{k}=\left[k_{x}, k_{x}, \frac{1}{2}\right]$, where $k_{x}$ equals $\frac{1}{4}$ for $R=\mathrm{Tb}, \mathrm{Er}$ and $\mathrm{Tm}$ and about 0.3 for $R=$ Ho. The impact of the crystalline electric field (CEF) manifests itself in a reduction of the rare earth magnetic moments in the ordered state, a change in the direction of the magnetic moments from parallel to the $c$ axis for $R=\mathrm{Tb}$ and Ho to perpendicular for $R=\mathrm{Er}$ and $\mathrm{Tm}$, and deviation from proportionality between the Néel temperature and the de Gennes factor. Therefore, the observed magnetic structures result from competition between indirect exchange interactions of the RKKY-type and the CEF.

\section{Acknowledgements}

Kind hospitality and financial support extended to two of us (SB and AS) by the Helmholtz-Zentrum Berlin für Materialien und Energie (HZB) is gratefully acknowledged. Our special thanks go to Professor Wiesława Sikora from the AGH University of Science and Technology (Kraków, Poland) for fruitful discussions of symmetry analysis of magnetic structures.

\section{Funding information}

This research was partially carried out with equipment purchased thanks to the financial support of the European Regional Development Fund in the framework of the Polish Innovation Economy Operational Program (contract No. POIG.02.01.00-12-023/08). The open-access publication of this article was funded by the Priority Research Area SciMat under the program "Excellence Initiative - Research University" at the Jagiellonian University in Krakow.

\section{References}

André, G., Thuéry, P., Pinot, M., Oleś, A. \& Szytuła, A. (1991). Solid State Commun. 80, 239-241. 
Baran, S., Kaczorowski, D., Arulraj, A., Penc, B. \& Szytuła, A. (2009). J. Magn. Magn. Mater. 321, 3256-3261.

Baran, S., Szytuła, A. \& Hoser, A. (2017). J. Alloys Compd. 696, 1278-1281.

De Gennes, P. (1962). J. Phys. Radium, 23, 510-521.

Diviš, M., Olšovec, M., Richter, M. \& Eschrig, H. (1995). J. Magn. Magn. Mater. 140-144, 1365-1366.

Fischer, P., Herrmannsdörfer, T., Bonelli, T., Fauth, F., Keller, L., Bauer, E. \& Giovannini, M. (2000). J. Phys. Condens. Matter, 12, 7089-7098.

Gondek, L., Przewoźnik, J., Czub, J., Tyvanchuk, Yu., Szytuła, A. \& Arurlaj, A. (2012). Intermetallics, 21, 10-17.

Hauser, R., Michor, H., Bauer, E., Hilscher, G. \& Kaczorowski, D. (1997). Physica B, 230-232, 211-213.

Kaczorowski, D., Rogl, P. \& Hiebl, K. (1996). Phys. Rev. B, 54, $9891-$ 9902.

Kalychak, Ya. M., Zaremba, V. I., Baranyak, V. M., Zavalii, P. Yu., Bruskov, V. A., Sysa, L. V. \& Dmytrakh, O. V. (1990). Izv. Akad. Nauk SSSR Neorg. Mater. 26, 94-96. [English translation: (1990). Inorg. Mater. 26, 74-76.]

Kalychak, Ya. M., Zaremba, V. I., Pöttgen, R., Lukachuk, M. \& Hoffmann, R.-D. (2004). Handbook on the Physics and Chemistry of Rare Earths, edited by K. A. Gschneidner Jr., J.-C. G. Bünzli \& V. K. Pecharsky, Vol. 34, ch. 218, pp. 1-133. Amsterdam: Elsevier.
Laffargue, D., Roisnel, T., Chevalier, B. \& Bourée, F. (1997). J. Alloys Compd. 262-263, 219-224.

Maskova, S., Danis, S., Llobet, A., Nakotte, H. \& Havela, L. (2014). Acta Phys. Pol. A, 126, 282-283.

Maslen, E. N., Streltsov, V. A. \& Ishizawa, N. (1996). Acta Cryst. B52, 414-422.

Matar, S. F. (1995). J. Magn. Magn. Mater. 151, 263-272.

Plumer, M. L. \& Caillé, A. (1992). Phys. Rev. B, 46, 203-206.

Rodríguez-Carvajal, J. (1993). Physica B, 192, 55-69.

Rodríguez-Carvajal, J. (2001). Newsletter of the Commission for Powder Diffraction of the IUCr, 26, 12-19.

Stevens, K. W. H. (1952). Proc. Phys. Soc. A, 65, 209-215.

Szytuła, A., Baran, S., Hoser, A., Kalychak, Ya. M., Penc, B. \& Tyvanchuk, Yu. (2013). Acta Phys. Pol. A, 124, 994-997.

Szytuła, A., Baran, S., Kaczorowski, D., Sikora, W. \& Hoser, A. (2014). J. Alloys Compd. 617, 149-153.

Szytuła, A., Baran, S., Przewoźnik, J., Tyvanchuk, Yu. \& Kalychak, Ya. (2015). J. Magn. Magn. Mater. 387, 83-89.

Tyvanchuk, Yu., Baran, S., Jaworska-Gołąb, T., Duraj, R., Kalychak, Ya. M. \& Szytuła, A. (2012). Acta Phys. Pol. A, 121, 678-681.

Zaremba, V. I., Bruskov, V. A., Zavalii, P. Yu. \& Kalychak, Ya. M. (1988). Izv. Akad. Nauk SSSR Neorg. Mater. 24, 409-411. [English translation: (1988). Inorg. Mater. 24, 330-332.]

Zhang, Z., Wang, P., Rong, H. \& Li, L. (2019). Dalton Trans. 48, 17792-17799. 